\title{
The diagnostic significance of breast incidentalomas detected on whole-body fluorine-18 fluorodeoxyglucose positron emission tomography/computed tomography
}

\begin{abstract}
Objective: The objective of this study was to establish the diagnostic significance of breast incidentalomas detected on whole-body fluorine-18 $\left({ }^{18} \mathrm{~F}\right)$ fluorodeoxyglucose (FDG) positron emission tomography/computed tomography (PET/CT).

Materials and Methods: We retrospectively analyzed the data of 3868 patients who underwent ${ }^{18} \mathrm{~F}$ FDG PET/CT at our institution, for the presence of hypermetabolic focus in the breasts. Patients with known breast cancer or with the previous history of breast cancer were excluded from the study. Ten out of remaining 3868 patients had abnormal focal uptake in the breast. We, therefore, enrolled these 10 patients with histopathology confirmation in this study.

Results: Among all 3868 patients, $10(0.25 \%)$ patients demonstrated incidental focal uptake in breast parenchyma. All of these 10 patients were females. Histopathology examination confirmed malignancy in 8 out of 10 patients (80\%), these included invasive ductal cancer in 4 patients, non-Hodgkin's lymphoma in 2 patients, and metastasis from rectal cancer and endometrial cancer, respectively, in 2 patients. Of the 10 patients, $2(20 \%)$ had lesions that were confirmed to be benign. Both of these were proven to be fibroadenomas. The mean maximum standardized uptake value $\left(\mathrm{SUV}_{\max }\right)$ on FDG-PET/CT scans was $1.35 \pm 1.2$ in the benign cases versus $3.8 \pm 1.83$ in the malignant cases. This difference was statistically insignificant $(P=0.056)$. All malignant lesions had SUV $\mathrm{max}_{2.0}$ or greater. The mean size differed significantly between the benign and malignant groups $(2.55 \pm 0.63$ vs. $1.31 \pm 0.44 \mathrm{~cm})(P=0.005)$ with benign lesions being bigger in size.

Conclusion: Unexpected focal areas of hypermetabolic activity discovered in the breast at the time of PET/CT are associated with a high likelihood of malignancy in as many as $80 \%$ of cases. Therefore, any suspicious activity discovered in the breast on PET/CT should be evaluated until a diagnosis is found.
\end{abstract}

Key words: Breast cancer; breast incidentaloma; breast lymphoma; breast metastases; positron emission tomography/computed tomography

\section{Introduction}

Positron emission tomography (PET) with fluorine-18 $\left({ }^{18} \mathrm{~F}\right)$ fluorodeoxyglucose (FDG) is widely used in oncology for diagnosis and staging of tumors and for monitoring the therapeutic response. Apart from providing metabolic information, these images provide good anatomic details as well. The widespread use of PET/computed tomography (CT) has increased the level of detection of incidental hypermetabolic foci unrelated to the known malignancy. The increased uptake may be associated with physiological or benign processes or due to the presence of additional primary or secondary

\begin{tabular}{|l|c|}
\hline \multicolumn{2}{|c|}{ Access this article online } \\
\hline \multirow{2}{*}{$\begin{array}{l}\text { Website: } \\
\text { www.asjo.in }\end{array}$} & Quick Response Code \\
\hline & \\
DOI: & \\
10.4103/2454-6798.173310 & \\
& \\
\hline
\end{tabular}

malignancies. ${ }^{[1]}$ The overall prevalence, by PET/CT, of incidental malignancies and premalignant pathology has been reported across literature to be $1.2-1.7 \%$; the most common sites involved being colon, thyroid, lung, and breast. ${ }^{[2-4]}$ These

\section{Shelly Sharma, Ankur Pruthi ${ }^{1}$}

Department of Radiology, Jaypee Hospital, Noida, Uttar Pradesh, ${ }^{1}$ Department of Nuclear Medicine, Sir Ganga Ram Hospital, New Delhi, India

Address for correspondence: Dr. Shelly Sharma, 2164, Block B/2, Vasant Kunj, New Delhi - 110 070, India. E-mail: raddocss@gmail.com

This is an open access article distributed under the terms of the Creative Commons Attribution-NonCommercial-ShareAlike 3.0 License, which allows others to remix, tweak, and build upon the work non-commercially, as long as the author is credited and the new creations are licensed under the identical terms.

For reprints contact: reprints@medknow.com

How to cite this article: Sharma S, Pruthi A. The diagnostic significance of breast incidentalomas detected on whole-body fluorine-18 fluorodeoxyglucose positron emission tomography/computed tomography. Asian J Oncol 2015;1:92-6. 
incidentally detected foci showing increased FDG uptake on $\mathrm{PET} / \mathrm{CT}$ can significantly impact patient outcome.

In women undergoing PET/CT for nonbreast malignancies, the incidence of unexpected increased ${ }^{18} \mathrm{~F}-\mathrm{FDG}$ activity within the breast tissue ranged from $0.36 \%$ to $6.3 \%$ and malignancy in these incidental hypermetabolic breast lesions ranged from $37.5 \%$ to $83 \%$. ${ }^{[5-8]}$ These studies demonstrated that incidental ${ }^{18} \mathrm{~F}$-FDG-avid lesions in the breast have a high incidence of malignancy and, therefore, need to have an appropriate evaluation. However, there are no clear-cut guidelines on evaluation of these lesions and often pose a diagnostic dilemma.

In this study, we retrospectively reviewed all the cases with incidentally detected focal breast lesions on PET/CT, who underwent PET/CT scan for nonbreast malignancies. In addition to performing quantitative analysis of the FDG uptake in the form of maximum standardized uptake value $\left(\mathrm{SUV}_{\max }\right)$, we also investigated whether noncontrast CT findings and mammography findings were helpful in characterizing these focal breast lesions.

\section{Materials and Methods}

\section{Subjects}

The study was performed in accordance with the regulations of the Institutional Review Board at our hospital, which approved this retrospective study and waived the requirement for patient informed consent. All patients who underwent ${ }^{18}$ F-FDG PET/CT scan between October 2012 and June 2013 at our institute were retrospectively analyzed. Patients with a known history of breast cancer and those with known breast lesions were excluded. Ten out of remaining 3868 patients had abnormal focal uptake in the breast. All these 10 patients had histopathology confirmation and were included in this study.

PET/CT-standard procedures for patient preparation and $\mathrm{PET} / \mathrm{CT}$ acquisition were followed. Patients were instructed to fast and not consume anything, except for water, for at least $4 \mathrm{~h}$ before the administration of ${ }^{18} \mathrm{~F}-\mathrm{FDG}$. Intravenous fluids containing dextrose were withheld for $4-6 \mathrm{~h}$ before tracer administration. Patients were administered $8-10 \mathrm{mCi}$ of ${ }^{18} \mathrm{~F}$-FDG intravenously provided blood glucose levels were $<150 \mathrm{mg} / \mathrm{dl}$. They were instructed to remain seated or recumbent after ${ }^{18} \mathrm{~F}$-FDG administration to avoid muscular uptake.

Image acquisition was done using Biograph 40 LSO advanced PET/CT scanner (Siemens, Knoxville, TN, USA). The system consists of a 40-slice, spiral CT (Siemens Somatom Emotion) and is optimized for use in whole-body oncology. Data were obtained in three-dimensional mode, with attenuation correction calculated from co-registered CT images. The $\mathrm{PET} / \mathrm{CT}$ scanner was subjected to daily quality control evaluation before the start of acquisition. Images were acquired 60 min after ${ }^{18} \mathrm{~F}$-FDG administration from skull base to mid-thigh (a transmission scan using CT followed by $2 \mathrm{~min}$ per bed emission scan).

\section{Image analysis}

All ${ }^{18} \mathrm{~F}$ FDG PET/CT scan images were interpreted by two experienced nuclear medicine physicians and a radiologist. Because of the physiologic distribution of FDG, uptake areas were identified as abnormal if the accumulation of FDG was focal and greater than in the background breast tissue. FDG uptake was measured in a semi-quantitative manner as $\mathrm{SUV}_{\max }$ corrected for body weight. The SUV is the decay-corrected ratio between the measured uptake in a region of interest, and the expected uptake if ${ }^{18} \mathrm{~F}$-FDG were distributed evenly throughout the body. A circular region of interest was placed over the region of highest intensity in the breast lesion, and uptake was automatically quantified as $\mathrm{SUV}_{\text {max }}$.

\section{Ultrasound and mammography}

An experienced radiologist later on performed a sonographic examination and the mammographic interpretation. All patients underwent breast sonography after PET/CT. Breast sonographic examination was performed with $7.5 \mathrm{MHz}$ probe (Sonosite Micromaxx). Mammographic examinations were performed in 6/10 patients on full field digital mammography unit (Selenia, Hologic).

\section{Results}

Among all 3868 patients, $10(0.25 \%)$ patients demonstrated incidental focal uptake in breast parenchyma. All of these 10 patients were females. None of these patients was a known case of carcinoma breast or any other breast pathology and were being evaluated for other malignancies. The mean age was $46 \pm 7$ (standard deviation [SD]) years in the group with benign lesions and $58.8 \pm 14.8$ (SD) years in the group with malignant lesions. This difference was statistically insignificant according to results of the Student's $t$-test $(P=0.1242)$. The mean $\mathrm{SUV}_{\text {max }}$ on FDG-PET/CT scans was $1.35 \pm 1.2$ in the benign cases versus $3.8 \pm 1.83$ in the malignant cases [Figure 1]. This difference was statistically insignificant $(P=0.056)$. All malignant lesions had $\mathrm{SUV}_{\max } 2.0$ or greater. The mean size differed significantly between the benign and malignant groups $(2.55 \pm 0.63$ vs. $1.31 \pm 0.44 \mathrm{~cm})$ $(P=0.005)$ with benign lesions being bigger in size [Table 1]. 


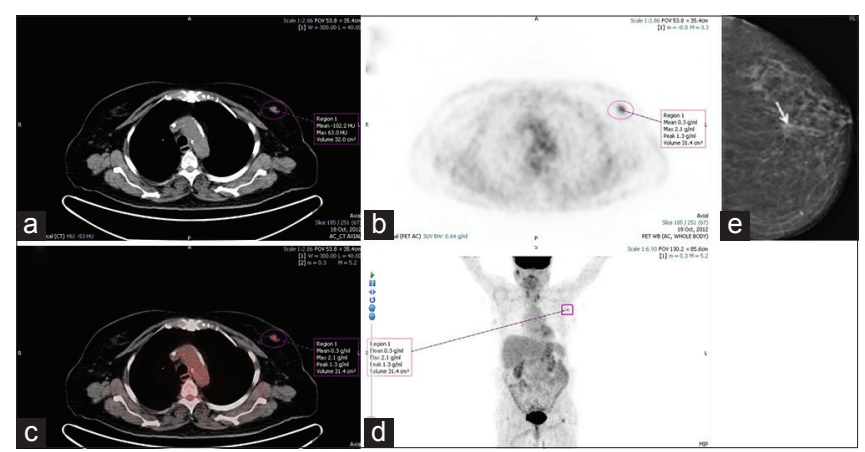

Figure 1: A 75-year-old woman (patient 1 in Table 1) with infiltrating ductal carcinoma was being evaluated for carcinoma of the ovary. (a) Computed tomography portion of fluorodeoxyglucose positron emission tomography/computed tomography shows a soft tissue lesion (measures $1.7 \mathrm{~cm} \times 1 \mathrm{~cm}$ ) with irregular margins. (b) Axial positron emission tomography image shows focal area of increased metabolism (maximum standardized uptake value $=2.1$ ) in the left breast, lateral to midline . (c) Positron emission tomography/computed tomography fused images and (d) positron emission tomography maximum intensity projection image. (e) Mammogram shows a soft tissue lesion (arrow) with spiculated margins corresponding to positron emission tomography/computed tomography abnormality

Table 1: Patient characteristics, standardized uptake values, and pathology results of unexpected hypermetabolic areas in the breast

\begin{tabular}{lcclc}
\hline $\begin{array}{l}\text { Underlying } \\
\text { malignancy }\end{array}$ & Age & Size & $\begin{array}{l}\text { Breast lesion } \\
\text { histopathology }\end{array}$ & SUV $_{\max }$ \\
\hline Ovary & 75 & 1.7 & Malignant & 2.0 \\
Lung & 41 & 3 & Benign & 2.2 \\
NHL (DLBCL) & 50 & 1 & Malignant & 3.1 \\
Rectum & 29 & 0.9 & Malignant & 3.3 \\
Ovary & 64 & 1 & Malignant & 2.8 \\
NHL & 74 & 0.8 & Malignant & 6.4 \\
Endometrium & 66 & 1.4 & Malignant & 6.6 \\
Periampullary & 56 & 1.7 & Malignant & 2 \\
Multiple myeloma & 57 & 2 & Malignant & 4.4 \\
Gall bladder & 51 & 2.1 & Benign & 0.5 \\
\hline
\end{tabular}

SUV - Standardized uptake values; DLBCL - Diffuse large B-cell lymphoma;

NHL - Non-Hodgkin's lymphoma

Histopathological examination confirmed malignancy in 8 out of 10 patients (80\%). Histopathological examination of specimens from these 8 patients revealed invasive ductal cancer in 4 patients, non-Hodgkin's lymphoma (NHL) in 2 patients, and metastasis from rectal cancer and endometrial cancer, respectively, in 2 patients. Of the 10 patients, $2(20 \%)$ had lesions that were confirmed to be benign and follow-up FDG PET/CT, and sonography showed no change [Figure 2]. Both of these were proven to be fibroadenomas.

Breast nodules were detected in the noncontrast CT portion of PET/CT in 9/10 cases. Of these, only two cases had irregular margins indicating high suspicion of malignancy. Both these cases were confirmed to be malignant. Rest of the nodules had well-defined or lobulated margins. In $1 / 10$ patients, the lesion was not detected on noncontrast CT

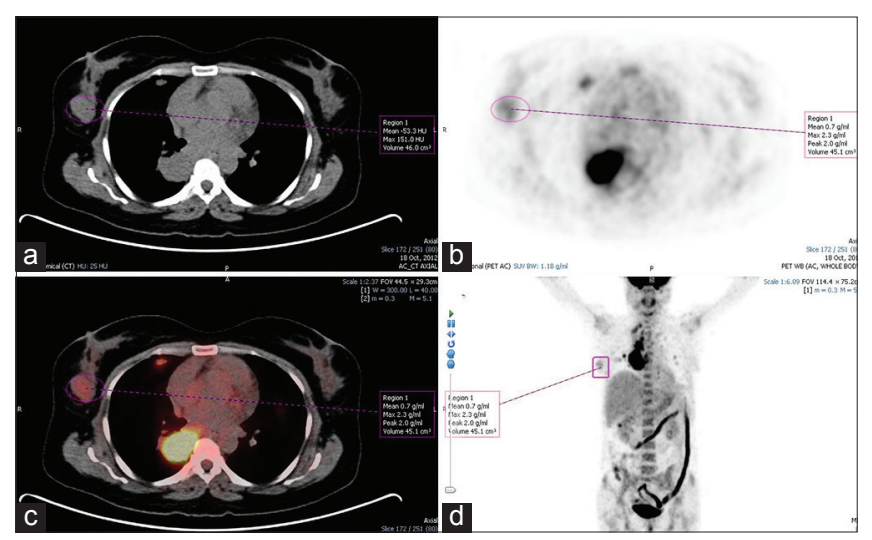

Figure 2: A 41-year-old woman (patient 2 in Table 1) with right breast fibroadenoma was being evaluated for carcinoma of the lung. (a) Computed tomography portion of fluorodeoxyglucose positron emission tomography/computed tomography shows a soft tissue lesion (measures $3.0 \mathrm{~cm} \times 1.6 \mathrm{~cm}$ ) in the outer quadrant with well-defined margins. (b) Axial positron emission tomography image shows focal area of increased metabolism (maximum standardized uptake value $=2.3$ ) in the right breast, outer quadrant. (c) Positron emission tomography/computed tomography fused image and (d) positron emission tomography-maximum intensity projection image

portion and showed diffuse FDG uptake in the lower inner quadrant of right breast [Figure 3]. Mammography was not available in all cases. Out of eight patients with malignant lesions, mammograms were available in six patients. The lesions were detected on mammography in $4 / 6$ patients. All of these patients had Breast Imaging-Reporting and Data System (BI-RADS) mammography Category IV or greater. In $2 / 6$ patients, the lesions were not detected on mammography due to dense breast parenchyma [Table 2].

\section{Discussion}

In this study, $10(0.25 \%)$ subjects demonstrated incidental focal uptake in the breast at FDG PET/CT. All of these patients were females. Eight of the 10 patients in the study were found to have malignant lesions. Thus, the frequency of malignancy was $80 \%$. Previously published reports have shown variable rates of malignancy in these incidental foci ranging between $37 \%$ and $83 \% .^{[5-8]}$ Our results matched with those reported by Korn et al. in a previously published similar study. ${ }^{[7]}$

In this study, we examined parameters that would be useful for differentiating the benign and malignant properties of incidentally identified FDG-avid foci in breasts. The malignant lesions were more common among older patients, but the difference between the two groups was not statistically significant $(P=0.12)$.

Second, all the malignant lesions had $\mathrm{SUV}_{\max } 2.0$ or greater and benign lesions showed low mean $\mathrm{SUV}_{\max }$. Similar studies in the past have found that SUV ${ }_{\max }$ can assist in differentiating 


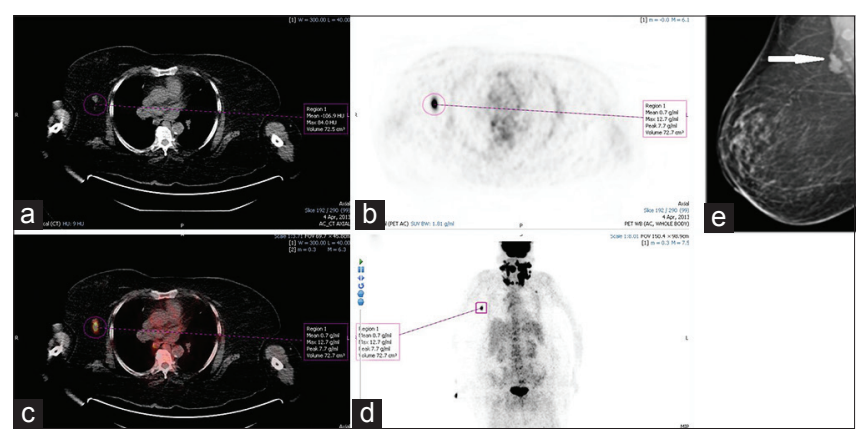

Figure 3: A 74-year-old woman (patient 6 in Table 1) with non-Hodgkin's lymphoma breast was being evaluated for non-Hodgkin's lymphoma of head and neck region. (a) Computed tomography portion of fluorodeoxyglucose positron emission tomography/computed tomography shows a soft tissue lesion (measuring $0.8 \mathrm{~cm}$ ) with regular margins. (b) Axial positron emission tomography image shows a hypermetabolic soft tissue nodule in right breast lower outer quadrant (maximum standardized uptake value $=12.7$ ). (c) Fused positron emission tomography/computed tomography images and (d) positron emission tomography-maximum intensity projection image. (e) Mammogram shows heterogeneously dense breast parenchyma with axillary lymph nodes (arrow)

between benign and malignant lesions..$^{[8-10]}$ Kang et al..$^{[8]}$ reported statistically significant difference in malignancy rate between the groups with $\mathrm{SUV}_{\text {max }}>2.0$ and $<2.0$. Our study did not find any statistical difference in $\mathrm{SUV}_{\text {max }}$ between benign and malignant lesions $(P=0.058)$, but mean $\mathrm{SUV}_{\max }$ in malignant lesions was higher than in benign lesions.

Third, the malignant lesions were smaller in size than the benign lesions. The difference between the two was statistically significant $(P=0.005)$. Chae et al..$^{[10]}$ in a similar study had found a statistically significant difference between the size of benign and malignant lesions with the diameter of malignant lesions being greater than that of benign lesions.

In this study, we excluded patients with previously known breast lesions and those with a history of breast cancer. Invasive ductal carcinoma (IDC) was the most common malignancy we encountered in this study (four of eight cases), and there were no cases of invasive lobular carcinoma. Invasive lobular carcinoma is reported to have a lower degree of FDG uptake than invasive ductal cancer and hence produces false negative results more often than IDCs at FDG $\mathrm{PET} / \mathrm{CT}^{[11,12]}$

In $2 / 8$ cases, there was extranodal involvement of breast by NHL. The $\mathrm{SUV}_{\text {max }}$ was $>2$ in both these cases. Almost any organ can be affected by lymphoma, with the most common extranodal sites of involvement being the stomach, spleen, waldeyer ring, central nervous system, lung, bone, and skin. ${ }^{[13]}$ Breast lymphoma is a rare disease representing $0.04-0.5 \%$ of malignant breast tumors and is almost always of non-Hodgkin's type. ${ }^{[14]}$ The breast involvement by lymphoma
Table 2: Imaging characteristics of malignant lesions on computed tomography scan

\begin{tabular}{lll}
\hline $\begin{array}{l}\text { Breast lesion } \\
\text { histopathology }\end{array}$ & $\begin{array}{l}\text { Detected } \\
\text { on CT scan }\end{array}$ & $\begin{array}{l}\text { Margins on } \\
\text { CT scan }\end{array}$ \\
\hline IDC & Yes & Irregular \\
NHL & Not detected & - \\
Metastatic & Yes & Smooth \\
IDC & Yes & Irregular \\
NHL & Yes & Regular \\
Metastatic & Yes & Regular \\
IDC & Yes & Lobulated \\
IDC & Yes & Lobulated \\
\hline NHL - Non-Hodgkin's lymphoma; CT - Computed tomography; IDC - Invasive ductal \\
carcinoma
\end{tabular}

can be subtle and may be overlooked during CT. PET/CT has evolved into an important imaging tool for evaluation of lymphomas, facilitating the detection of affected extranodal sites even when CT shows subtle or no obvious lesions. ${ }^{[15]}$

In $2 / 8$ cases, there were metastases to the breast from known primary sites. Initially, these lesions were presumed to be primary breast malignancies; however, immunohistochemical studies confirmed them to be metastasis from rectal and endometrial cancer, respectively. Hematogenous spread of metastases to the breast is rare with the most common malignancies to metastasize to the breast being melanoma, sarcoma, and tumors of the lung and ovary. ${ }^{[16,17]}$ The differentiation between a primary breast cancer and a metastatic lesion on the basis of imaging characteristics can be difficult. Patients with metastatic disease to the breast usually present with one or more discrete, well-circumscribed nodules that may be similar to benign nodules. Internal calcifications are rare in metastatic nodules, except in the case of metastatic ovarian carcinoma, in which the calcifications represent psammoma bodies. ${ }^{[16]}$ In addition, spiculation is uncommon because of the absence of desmoplasia in these lesions. ${ }^{[17]}$ In both our cases, the lesions had smooth margins with no associated micro calcifications.

The noncontrast CT portion of PET/CT detected 9/10 lesions. The characteristic imaging features of malignancy were present in only $2 / 8$ malignant lesions. Six of these eight patients with confirmed malignancy underwent concomitant mammography, and mass lesions were detected in four of these six cases. All of these four patients had mammography BI-RADS Category IV or higher. Thus, we did not find these modalities helpful in characterizing these lesions into benign or malignant categories.

However, all the lesions (10/10) were identified on ultrasound which was also used as a guide for tissue sampling. The specificity of mammography was found to be lower than that of 
sonography; the reason for this can be dense breast parenchyma and also the smaller size of these lesions. ${ }^{[18]}$ Ultrasound is an easily available modality and can be used as an adjunct to PET/CT for evaluation and characterization of these lesions.

Our study revealed that incidental focal breast uptake of $\mathrm{SUV}_{\max } 2.0$ or greater is significant and requires further evaluation. However, the need for further workup should be determined by clinical judgment and extent of primary disease. Beatty et al. ${ }^{[2]}$ prospectively acquired a database of patients with a known malignancy that underwent PET/CT for staging or serial imaging. They concluded that incidental PET/CT findings should be investigated when the results will impact treatment.

The limitations of this study are its retrospective nature and a small study group.

\section{Conclusion}

Unexpected focal areas of hypermetabolic activity discovered in the breast at the time of PET/CT are associated with a high likelihood of malignancy in as many as $80 \%$ of cases. Therefore, any suspicious activity discovered in the breast on PET/CT should be evaluated until a diagnosis is found.

\section{Financial support and sponsorship}

Nil.

\section{Conflicts of interest}

There are no conflicts of interest.

\section{References}

1. Clapp AJ, Peller PJ, Subramaniam RM. AJR teaching file: Incidental breast cancer detected with 18F-FDG PET/CT. AJR Am J Roentgenol 2011;196 6 Suppl: WS83-5.

2. Beatty JS, Williams HT, Aldridge BA, Hughes MP, Vasudeva VS, Gucwa AL, et al. Incidental PET/CT findings in the cancer patient: How should they be managed? Surgery 2009;146:274-81.
3. Ishimori T, Patel PV, Wahl RL. Detection of unexpected additional primary malignancies with PET/CT. J Nucl Med 2005;46:752-7.

4. Agress H Jr., Cooper BZ. Detection of clinically unexpected malignant and premalignant tumors with whole-body FDG PET: Histopathologic comparison. Radiology 2004;230:417-22.

5. Beatty JS, Williams HT, Gucwa AL, Hughes MP, Vasudeva VS, Aldridge BA, et al. The predictive value of incidental PET/CT findings suspicious for breast cancer in women with non-breast malignancies. Am J Surg 2009;198:495-9.

6. Litmanovich D, Gourevich K, Israel O, Gallimidi Z. Unexpected foci of $18 \mathrm{~F}-\mathrm{FDG}$ uptake in the breast detected by PET/CT: Incidence and clinical significance. Eur J Nucl Med Mol Imaging 2009;36:1558-64.

7. Korn RL, Yost AM, May CC, Kovalsky ER, Orth KM, Layton TA, et al. Unexpected focal hypermetabolic activity in the breast: Significance in patients undergoing 18F-FDG PET/CT. AJR Am J Roentgenol 2006;187:81-5.

8. Kang BJ, Lee JH, Yoo IeR, Kim SH, Choi JJ, Jeong SH, et al. Clinical significance of incidental finding of focal activity in the breast at 18F-FDG PET/CT. AJR Am J Roentgenol 2011;197:341-7.

9. Dunne RM, O'Mahony D, Wilson G, McDermott R, O'Keeffe SA. The role of the breast radiologist in evaluation of breast incidentalomas detected on 18-fludeoxyglucose positron emission tomography/CT. Br J Radiol 2013;86:20130034.

10. Chae EY, Cha JH, Kim HH, Shin HJ, Kim HJ, Oh HY, et al. Analysis of incidental focal hypermetabolic uptake in the breast as detected by 18F-FDG PET/CT: Clinical significance and differential diagnosis. Acta Radiol 2012;53:530-5.

11. Avril N, Adler LP. F-18 fluorodeoxyglucose-positron emission tomography imaging for primary breast cancer and loco-regional staging. Radiol Clin North Am 2007;45:645-57, vi.

12. Buck A, Schirrmeister H, Kühn T, Shen C, Kalker T, Kotzerke J, et al. FDG uptake in breast cancer: Correlation with biological and clinical prognostic parameters. Eur J Nucl Med Mol Imaging 2002;29:1317-23.

13. Paes FM, Kalkanis DG, Sideras PA, Serafini AN. FDG PET/CT of extranodal involvement in non-Hodgkin lymphoma and Hodgkin disease. Radiographics 2010;30:269-91.

14. Garg NK, Bagul NB, Rubin G, Shah EF. Primary lymphoma of the breast involving both axillae with bilateral breast carcinoma. World J Surg Oncol 2008;6:52.

15. Ilica AT, Kocacelebi K, Savas R, Ayan A. Imaging of extranodal lymphoma with PET/CT. Clin Nucl Med 2011;36:E127-38.

16. Paulus DD, Libshitz HI. Metastasis to the breast. Radiol Clin North Am 1982;20:561-8.

17. McCrea ES, Johnston C, Haney PJ. Metastases to the breast. AJR Am J Roentgenol 1983;141:685-90.

18. Kolb TM, Lichy J, Newhouse JH. Occult cancer in women with dense breasts: Detection with screening US - Diagnostic yield and tumor characteristics. Radiology 1998;207:191-9. 